\title{
Interactive Annotation Learning with Indirect Feature Voting
}

\author{
Shilpa Arora and Eric Nyberg \\ Language Technologies Institute \\ Carnegie Mellon University \\ Pittsburgh, PA 15213, USA \\ \{shilpaa,ehn\}@es.cmu.edu
}

\begin{abstract}
We demonstrate that a supervised annotation learning approach using structured features derived from tokens and prior annotations performs better than a bag of words approach. We present a general graph representation for automatically deriving these features from labeled data. Automatic feature selection based on class association scores requires a large amount of labeled data and direct voting can be difficult and error-prone for structured features, even for language specialists. We show that highlighted rationales from the user can be used for indirect feature voting and same performance can be achieved with less labeled data.We present our results on two annotation learning tasks for opinion mining from product and movie reviews.
\end{abstract}

\section{Introduction}

Interactive Annotation Learning is a supervised approach to learning annotations with the goal of minimizing the total annotation cost. In this work, we demonstrate that with additional supervision per example, such as distinguishing discriminant features, same performance can be achieved with less annotated data. Supervision for simple features has been explored in the literature (Raghavan et al., 2006; Druck et al., 2008; Haghighi and Klein, 2006). In this work, we propose an approach that seeks supervision from the user on structured features.

Features that capture the linguistic structure in text such as n-grams and syntactic patterns, referred to as structured features in this work, have been found to be useful for supervised learning of annotations. For example, Pradhan et al. (2004) show that using features like syntactic path from constituent to predicate improves performance of a semantic parser. However, often such features are "handcrafted" by domain experts and do not generalize to other tasks and domains. In this work, we propose a general graph representation for automatically extracting structured features from tokens and prior annotations such as part of speech, dependency triples, etc. Gamon (2004) shows that an approach using a large set of structured features and a feature selection procedure performs better than an approach that uses a few "handcrafted" features. Our hypothesis is that structured features are important for supervised annotation learning and can be automatically derived from tokens and prior annotations. We test our hypothesis and present our results for opinion mining from product reviews.

Deriving features from the annotation graph gives us a large number of very sparse features. Feature selection based on class association scores such as mutual information and chi-square have often been used to identify the most discriminant features (Manning et al., 2008). However, these scores are calculated from labeled data and they are not very meaningful when the dataset is small. Supervised feature selection, i.e. asking the user to vote for the most discriminant features, has been used as an alternative when the training dataset is small. Raghavan et al. (2006) and Druck et al. (2008) seek feedback on unigram features from the user for document classification tasks. Haghighi and Klein (2006) ask the user to suggest a few prototypes (examples) for each class and use those as features. These approaches ask the annotators to identify globally rel- 
evant features, but certain features are difficult to vote on without the context and may take on very different meanings in different contexts. Also, all these approaches have been demonstrated for unigram features and it is not clear how they can be extended straightforwardly to structured features.

We propose an indirect approach to interactive feature selection that makes use of highlighted rationales from the user. A rationale (Zaidan et al., 2007) is the span of text a user highlights in support of his/her annotation. Rationales also allow us to seek feedback on features in context. Our hypothesis is that with rationales, we can achieve same performance with lower annotation cost and we demonstrate this for opinion mining from movie reviews.

In Section 2, we describe the annotation graph representation and motivate the use of structured features with results on learning opinions from product reviews. In Section 3, we show how rationales can be used for identifying the most discriminant features for opinion classification with less training data. We then list the conclusions we can draw from this work, followed by suggestions for future work.

\section{Learning with Structured Features}

In this section, we demonstrate that structured features help in improving performance and propose a formal graph representation for deriving these features automatically.

\subsection{Opinions and Structured Features}

Unigram features such as tokens are not sufficient for recognizing all kinds of opinions. For example, a unigram feature good may seem useful for identifying opinions, however, consider the following two comments in a review: 1) This camera has good features and 2) I did a good month's worth of research before buying this camera. In the first example, the unigram good is a useful feature. However, in the second example, good is not complementing the camera and hence will mislead the classifier. Structured features such as part-of-speech, dependency relations etc. are needed to capture the language structure that unigram features fail to capture.

\subsection{Annotation Graph and Features}

We define the annotation graph as a quadruple: $G=$ $(N, E, \Sigma, \lambda)$, where $N$ is the set of nodes, $E$ is the set of edges $E \subset N \times N, \Sigma=\Sigma_{N} \cup \Sigma_{E}$ is a set of labels for nodes and edges. $\lambda$ is the labeling function $\lambda: N \cup E \rightarrow \Sigma$, that assigns labels to nodes and edges. In this work, we define the set of labels for nodes, $\Sigma_{N}$ as tokens, part of speech and dependency annotations and set of labels for edges, $\Sigma_{E}$ as relations, $\Sigma_{E}=\{$ leftOf, parentOf, restricts $\}$. The left $O f$ relation is defined between two adjacent nodes. The parentof relation is defined between the dependency type and its attributes. For example, for the dependency triple 'nsubj_perfect_camera', there is a parent $O f$ relation between the dependency type 'nsubj' and tokens 'perfect' and 'camera'. The restricts relation exists between two nodes $a$ and $b$ if their textual spans overlap completely and $a$ restricts how $b$ is interpreted. For a word with multiple senses the restricts relation between the word and its part of speech, restricts the way the word is interpreted, by capturing the sense of the word in the given context. The Stanford POS tagger (Toutanova and Manning, 2000) and the Stanford parser (Klein and Manning, 2003) were used to produce the part of speech and dependency annotations.

Features are defined as subgraphs, $G^{\prime}=$ $\left(N^{\prime}, E^{\prime}, \Sigma^{\prime}, \lambda^{\prime}\right)$ in the annotation graph $G$, such that $N^{\prime} \subseteq N, E^{\prime} \subset N^{\prime} \times N^{\prime}$ and $E^{\prime} \subseteq E, \Sigma^{\prime}=\Sigma_{N}^{\prime} \cup \Sigma_{E}^{\prime}$ where $\Sigma_{N}^{\prime} \subseteq \Sigma_{N}$ and $\Sigma_{E}^{\prime} \subseteq \Sigma_{E}$ and $\lambda^{\prime}: N^{\prime} \cup E^{\prime} \rightarrow$ $\Sigma^{\prime}$. For a bag of words approach that only uses tokens as features, $\Sigma_{N}^{\prime}=T$, where $T$ is the token vocabulary and $E=\phi$ and $\Sigma_{E}=\phi$ (where $\phi$ is the null set). We define the degree of a feature subgraph as the number of edges it contains. For example, the unigram features are the feature subgraphs with no edges i.e. degree $=0$. Degree -1 features are the feature subgraphs with two nodes and an edge. In this paper, we present results for feature subgraphs with degree $=0$ and degree $=1$.

Figure 1 shows the partial annotation graph for two comments discussed above. The feature subgraph that captures the opinion expressed in 1(a), can be described in simple words as "camera has features that are good". This kind of subject-object relationship with the same verb, between the 'camera' and what's being modified by 'good', is not present in the second example (1(b)). A slight modification of 1(b), I did a month's worth of research before buying this good camera does express an opinion about the camera. A bag of words approach that uses only unigram features will not be able to differ- 
entiate between these two examples; structured features like dependency relation subgraphs can capture this linguistic distinction between the two examples.

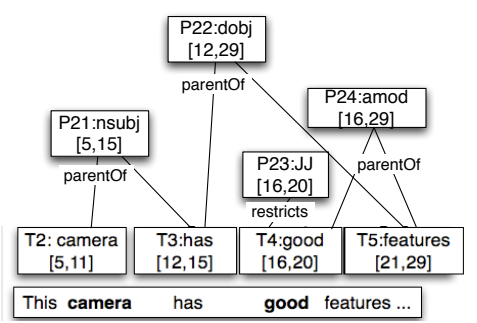

(a)

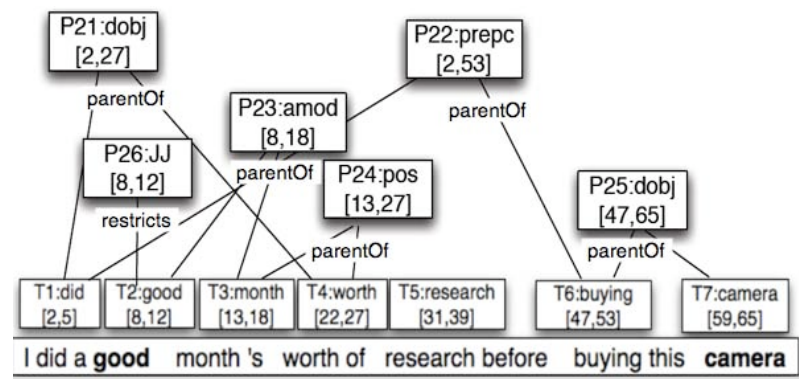

(b)

Figure 1: The figure shows partial annotation graphs for two examples. Only some of the nodes and edges are shown for clarity. Spans of nodes in brackets are the character spans.

\subsection{Experiments and Results}

The dataset we used is a collection of 244 Amazon's customer reviews (2962 comments) for five products (Hu and Liu, 2004). A review comment is annotated as an opinion if it expresses an opinion about an aspect of the product and the aspect is explicitly mentioned in the sentence. We performed 10-fold cross validation $(\mathrm{CV})$ using the Support Vector Machine (SVM) classifier in MinorThird (Cohen, 2004) with the default linear kernel and chi-square feature selection to select the top 5000 features. As can be seen in Table 1, an approach using degree - 0 features, i.e. unigrams, part of speech and dependency triples together, outperforms using any of those features alone and this difference is significant. Using degree -1 features with two nodes and an edge improves performance further. However, using degree -0 features in addition to degree -1 features does not improve performance. This suggests that when using higher degree features, we may leave out the features with lower degree that they subsume.

\begin{tabular}{|l|l|l|}
\hline Features & Avg F1 & Outperforms \\
\hline unigram [uni] & 65.74 & pos,dep \\
\hline pos-unigram [pos] & 64 & dep \\
\hline dependency [dep] & 63.18 & - \\
\hline degree-0 [deg-0] & 67.77 & uni,pos,dep \\
\hline degree-1 [deg-1] & $\mathbf{7 0 . 5 6}$ & uni,pos,dep,deg-0, deg-* \\
\hline (deg-0 + deg-1) [deg-*] & 70.12 & uni,pos,dep,deg-0 \\
\hline
\end{tabular}

Table 1: The table reports the F-measure scores averaged over ten cross validation folds. The value in bold in the Avg F1 column is the best performing feature combination. For each feature combination in the row, outperforms column lists the feature combinations it outperforms, with significant differences highlighted in bold (paired t-test with $p<$ 0.05 considered significant).

\section{Rationales \& Indirect Feature voting}

We propose an indirect feature voting approach that uses user-highlighted rationales to identify the most discriminant features. We present our results on Movie Review data annotated with rationales.

\subsection{Data and Experimental Setup}

The data set by Pang and Lee (2004) consists of 2000 movie reviews (1000-pos, 1000-neg) from the IMDb review archive. Zaidan et al. (2007) provide rationales for 1800 reviews (900-pos, 900-neg). The annotation guidelines for marking rationales are described in (Zaidan et al., 2007). An example of a rationale is: "the movie is so badly put together that even the most casual viewer may notice the miserable pacing and stray plot threads". For a test dataset of 200 reviews, randomly selected from 1800 reviews, we varied the training data size from 50 to 500 reviews, adding 50 reviews at a time. Training examples were randomly selected from the remaining 1600 reviews. During testing, information about rationales is not used.

We used tokens ${ }^{1}$, part of speech and dependency triples as features. We used the KStem stemmer (Krovetz, 1993) to stem the token features. In order to compare the approaches at their best performing feature configuration, we varied the total number of features used, choosing from the set: $\{1000$, $2000,5000,10000,50000\}$. We used chi-square feature selection (Manning et al., 2008) and the SVM learner with default settings from the Minorthird package (Cohen, 2004) for these experiments. We compare the following approaches:

Base Training Dataset $(B T D)$ : We train a model from the labeled data with no feature voting.

\footnotetext{
${ }^{1}$ filtering the stop words using the stop word list: http: //www.cs.cmu.edu/ shilpaa/stop-words-ial-movie. txt
} 
Rationale annotated Training Dataset ( $R T D)$ : We experimented with two different settings for indirect feature voting: 1) only using features that overlap with rationales $(R T D(1,0)) ; 2)$ features from rationales weighted twice as much as features from other parts of the text $(R T D(2,1))$. In general, $R(i, j)$ describes an experimental condition where features from rationales are weighted $i$ times and other features are weighted $j$ times. In Minorthird, weighing a feature two times more than other features is equivalent to that feature occurring twice as much.

Oracle voted Training Data (OTD): In order to compare indirect feature voting to direct voting on features, we simulate the user's vote on the features with class association scores from a large dataset (all 1600 documents used for selecting training documents). This is based on the assumption that the class association scores, such as chi-square, from a large dataset can be used as a reliable discriminator of the most relevant features. This approach of simulating the oracle with large amount of labeled data has been used previously in feature voting (Raghavan et al., 2006).

\subsection{Results and Discussion}

In Table 2, we present the accuracy results for the four approaches described in the previous section. We compare the best performing feature configurations for three approaches - BTD, RTD $(1,0)$ and $R T D(2,0)$. As can be seen, $R T D(1,0)$ always performs better than $B T D$. As expected, improvement with rationales is greater and it is significant when the training dataset is small. The performance of all approaches converge as the training data size increases and hence we only present results up to training dataset size of 500 examples in this paper.

Since our goal is to evaluate the use of rationales independently of how many features the model uses, we also compared the four approaches in terms of the accuracy averaged over five feature configurations. Due to space constraints, we do not include the table of results. On average $R T D(1,0)$ significantly outperforms $B T D$ when the total training dataset is less than 350 examples. When the training data has fewer than 400 examples, $R T D(1,0)$ also significantly outperforms $R T D(2,1)$.

$O T D$ with simulated user is an approximate up-

\begin{tabular}{|c|c|c|c|c|c|c|}
\hline \multirow{2}{*}{ \#Ex } & \multirow{2}{*}{ Approach } & \multicolumn{5}{|c|}{ Number of Features } \\
\hline & & 1000 & 2000 & 5000 & 10000 & 50000 \\
\hline \multirow{4}{*}{50} & OTD & 67.63 & 66.30 & 62.90 & 52.17 & 55.03 \\
\hline & BTD & $\mathbf{5 8 . 1 0}$ & 57.47 & 52.67 & 51.80 & 55.03 \\
\hline & RTD(1, & 55.43 & 55.93 & 61.63 & 61.63 & 61.63 \\
\hline & $\operatorname{RTD}(2$, & 57.77 & 57.53 & 52.73 & 52.30 & 56.33 \\
\hline \multirow{4}{*}{100} & OTD & 71.97 & 71.07 & 70.27 & 69.37 & 64.33 \\
\hline & BTD & 64.17 & 64.43 & 62.70 & 56.63 & 64.37 \\
\hline & $\operatorname{RTD}(1,0) *$ & 65.43 & 63.27 & 65.13 & 67.23 & 67.23 \\
\hline & $\operatorname{RTD}(2$, & 64.27 & 63.93 & 62.47 & 56.10 & 63.77 \\
\hline \multirow{4}{*}{150} & OTD & 73.83 & 74.83 & 74.20 & 74.00 & 63.83 \\
\hline & BTD & 66.17 & 67.77 & 68.60 & 64.33 & 60.47 \\
\hline & $\operatorname{RTD}(1,0) *$ & 69.30 & 68.30 & 67.27 & 71.30 & 71.87 \\
\hline & RTD $(2$ & 68.00 & 67.07 & 68.43 & 63.57 & 58.90 \\
\hline \multirow{4}{*}{200} & OTD & 74.83 & 75.87 & 75.70 & 75.10 & 56.97 \\
\hline & BTD & 71.63 & 71.37 & 72.57 & 71.53 & 58.90 \\
\hline & $\operatorname{RTD}(1,0)$ & 72.23 & 72.63 & 71.63 & 73.80 & 73.93 \\
\hline & $\operatorname{RTD}(2$, & 71.20 & 71.10 & 73.03 & 70.77 & 57.87 \\
\hline \multirow{4}{*}{250} & OTD & 75.63 & 76.90 & 77.70 & 77.67 & 62.20 \\
\hline & BTD & 72.60 & 73.57 & 74.73 & 75.20 & 58.93 \\
\hline & $\operatorname{RTD}(1,0)$ & 73.00 & 73.57 & 73.57 & 74.70 & 76.70 \\
\hline & $\operatorname{RTD}(2$ & 72.87 & 73.90 & 74.63 & 75.40 & 57.43 \\
\hline \multirow{4}{*}{300} & OTD & 76.57 & 77.67 & 78.93 & 78.43 & 68.17 \\
\hline & BTD & 72.97 & 74.13 & 74.93 & 76.57 & 63.83 \\
\hline & RTD(1) & 74.43 & 74.83 & 74.67 & 74.73 & 77.67 \\
\hline & $\operatorname{RTD}(2$ & 72.67 & 74.53 & 74.37 & 76.53 & 61.30 \\
\hline \multirow{4}{*}{350} & OTD & 76.47 & 78.20 & 80.20 & 79.80 & 71.73 \\
\hline & BTD & 74.43 & 74.30 & 74.73 & 77.27 & 66.80 \\
\hline & $\operatorname{RTD}(1,0)$ & 75.07 & 76.20 & 75.80 & 75.20 & 78.53 \\
\hline & $\operatorname{RTD}(2$, & 74.63 & 75.70 & 74.80 & 78.23 & 64.93 \\
\hline \multirow{4}{*}{400} & OTD & 77.97 & 78.93 & 80.53 & 80.60 & 75.27 \\
\hline & BTD & 75.83 & 76.77 & 76.47 & 78.93 & 70.63 \\
\hline & RTD(1 & 75.17 & 76.40 & 75.83 & 76.00 & 79.23 \\
\hline & $\mathrm{RTD}(2$ & 75.73 & 76.07 & 76.80 & 78.50 & 68.20 \\
\hline \multirow{4}{*}{450} & OTD & 77.67 & 79.20 & 80.57 & 80.73 & 77.13 \\
\hline & BTD & 75.73 & 76.80 & 77.80 & 78.80 & 74.37 \\
\hline & $\operatorname{RTD}(1,0)$ & 74.83 & 76.50 & 76.23 & 76.47 & 80.40 \\
\hline & $\mathrm{RTD}(2$ & 75.87 & 76.87 & 77.87 & 78.87 & 71.80 \\
\hline \multirow{4}{*}{500} & OTD & 78.03 & 80.10 & 81.27 & 81.67 & 79.87 \\
\hline & BTD & 75.27 & 77.33 & 79.37 & 80.30 & 75.73 \\
\hline & RTD(1 & 75.77 & 77.63 & 77.47 & 77.27 & 81.10 \\
\hline & $\operatorname{RTD}(2,1)$ & 75.83 & 77.47 & 79.50 & 79.70 & 74.50 \\
\hline
\end{tabular}

Table 2: Accuracy performance for four approaches, five feature configurations and increasing training dataset size. Accuracy reported is averaged over five random selection of training documents for three randomly selected test datasets. The numbers in bold in a row represents the best performing feature configuration for a given approach and training dataset size. The approach in bold represents the best performing approach among $B T D, R T D(1,0)$ and $R T D(2,1)$ for a given training dataset size. ' $*$ ' indicates significant improvement in performance over $B T D$ (paired t-test with $p<0.05$ considered significant).

per bound for rationale based approaches. It tells us how far we are from direct supervision on structured features. On average, OTD significantly outperformed $R T D(1,0)$ for training data size of 100 , $150,400,450$ and 500 examples but not always. As can be seen from Table 2, difference between $O T D$ and $R T D(1,0)$ reduces with more training data, since with more data and hence more rationales we get better feature coverage.

Results presented here show that for a given training dataset, we can boost the performance by ask- 
ing the user to label rationales. However, there is an additional cost associated with the rationales. It is important to evaluate how much total annotation cost rationales can save us while achieving the desired performance. In Figure 2, we compare the number of training examples an approach needs to achieve a given level of performance. As can be seen, $R T D(1,0)$ needs fewer training examples to achieve the same performance as $B T D$. The difference is large initially when the total number of training examples is small $(50$ for $R T D(1,0)$ and 150 for $B T D$ to achieve a performance between $66-67)$.

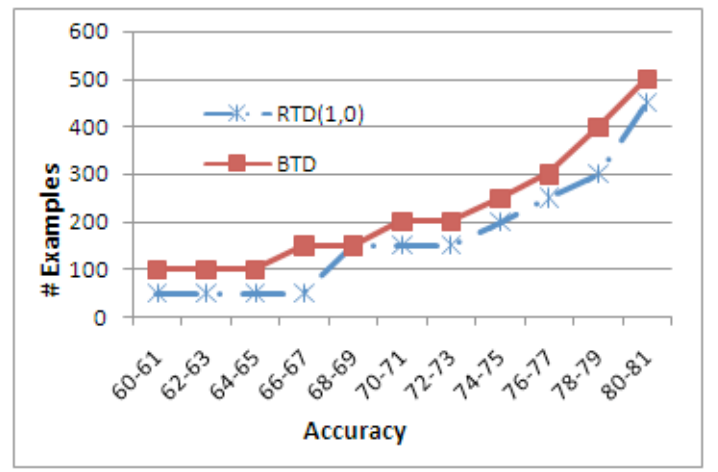

Figure 2: The Figure shows the number of examples needed by the two approaches, $R T D(1,0)$ and $B T D$, to achieve an accuracy in the given range.

Comparison with Zaidan et al. (2007): Zaidan et al. (2007) conclude that using only features from rationales performs worse than both: 1) using all the features in the documents, and 2) using features that do not overlap with the rationales. The results presented in this paper seem to contradict their results. However, they only experimented with unigram features and only one approach to using features from rationales, $R T D(1,0)$ and not $R T D(2,1)$. In order to compare our work directly with theirs, we experimented with an equivalent set of unigram features. In Table 3, we present the results using same number of total features (17744) as Zaidan et al. (2007). As can be seen from the table, when only unigram features are used, $R T D(2,1)$ outperforms $B T D$ but $R T D(1,0)$ performs worse than $B T D$. Thus, our results are consistent with (Zaidan et al., 2007) i.e. using unigram features only from the rationales does not boost performance.

From Table 3, we also analyze the improvement in performance when part of speech and dependency features are used in addition to the unigram features i.e. using all degree -0 subgraph fea-

\begin{tabular}{|l|l|l|l|l|}
\hline \#Ex & Approach & uni & uni-pos & uni-pos-dep \\
\hline \multirow{5}{*}{100} & OTD & 68.6 & 68.8 & 61.6 \\
& BTD & 68.6 & 68.8 & 52.2 \\
& RTD(1,0) & 68.2 & 68.1 & $\mathbf{6 9 . 0} *$ \\
& RTD(2,0) & $\mathbf{7 0 . 0}$ & 67.0 & 51.7 \\
\hline \multirow{5}{*}{200} & OTD & 73.6 & 73.8 & 75.3 \\
& BTD & 73.6 & $\mathbf{7 3 . 8}$ & 67.1 \\
& RTD(1,0) & 73.9 & 73.2 & $\mathbf{7 3 . 9} *$ \\
& RTD(2,0) & $\mathbf{7 5 . 3} *$ & 70.3 & 65.2 \\
\hline \multirow{5}{*}{300} & OTD & 76.2 & 76.1 & 79.1 \\
& BTD & 76.2 & $\mathbf{7 6 . 1}$ & 73.7 \\
& RTD $(1,0)$ & 75.0 & 74.9 & $\mathbf{7 7 . 1}^{*}$ \\
& RTD(2,0) & $\mathbf{7 7 . 5 *}$ & 73.3 & 74.8 \\
\hline \multirow{5}{*}{400} & OTD & 77.4 & 76.8 & 79.9 \\
& BTD & 77.4 & $\mathbf{7 6 . 8}$ & 76.2 \\
& RTD(1,0) & 75.9 & 75.9 & 77.0 \\
& RTD(2,0) & $\mathbf{7 8 . 0}$ & 74.7 & $\mathbf{7 7 . 7 *}$ \\
\hline \multirow{5}{*}{500} & OTD & 78.1 & 78.1 & 80.0 \\
& BTD & 78.1 & $\mathbf{7 8 . 1}$ & 78.4 \\
& RTD(1,0) & 76.3 & 76.2 & 77.6 \\
& RTD(2,0) & $\mathbf{7 8 . 2}$ & 75.4 & $\mathbf{7 9 . 0}$ \\
\hline
\end{tabular}

Table 3: The Table reports accuracy for four approaches in a setting similar to (Zaidan et al., 2007). Accuracy reported is averaged over ten random selection of training documents for two randomly selected test datasets.The numbers in bold are the best among $B T D, R T D(1,0)$, $R T D(2,1)$ for a given feature combination. ' $*$ ' highlights the significant improvement in performance over BTD (using paired t-test, with $p<0.05$ considered significant).

tures. For $R T D(1,0)$, adding these features improves performance for all data sizes with significant improvement for dataset size of 300 and $500 \mathrm{ex}-$ amples. $R T D(1,0)$ also significantly outperforms $B T D$ when all three features are used. For direct voting on features $(O T D)$, a significant improvement with these structured features is seen when the training dataset size is greater than 200 examples. For $B T D$ and $R T D(2,1)$ approaches, there is no significant improvement with these additional features. In the future, we plan to investigate further the benefit of using higher degree subgraph features for opinion mining from the movie review data.

Comparing ranking of features:We also compared the features that the rationales capture to what the oracle will vote for as the most relevant features. Features are ranked based on chi-square scores used in feature selection. We compare the ranked list of features from $R T D(1,0), B T D$ and $O T D$ and use a weighted F-measure score for evaluating the top 100 ranked features by each approach. This measure is inspired by the Pyramid measure used in Summarization (Nenkova and Passonneau, 2004). Instead of using counts in calculating F-measure, we used the chi-square score assigned to the features by the oracle dataset, in order to give more weight to the more discriminant features. As can be seen from 
Table $4, R T D(1,0)$ outperforms $B T D$ in capturing the important features when the datasize set is small $(<300)$ and this difference is significant. Beyond 300 examples, as the data size increases, $B T D$ outperforms $R T D(1,0)$. This implies that the rationales alone are able to capture the most relevant features when the dataset is small.

\begin{tabular}{|l||l|l|l|l|l|l|l|}
\hline & 100 & 200 & 300 & 400 & 500 & 600 & 700 \\
\hline RO & $\mathbf{4 7 . 7 0}$ & $\mathbf{5 3 . 8 0}$ & $\mathbf{5 7 . 6 8}$ & 59.54 & 62.13 & 60.86 & 61.56 \\
\hline TO & 31.22 & 44.43 & 52.98 & 60.57 & 64.61 & $\mathbf{6 7 . 1 0}$ & $\mathbf{7 0 . 3 9}$ \\
\hline
\end{tabular}

Table 4: Weighted F-measure performance comparison of ranked list of features from $R T D(1,0) \& O T D(R O)$ and $B T D \& O T D(T O)$. Results are averaged over ten random selections of the training data for a randomly selected test dataset. Significant differences are highlighted in bold (paired t-test with $p<0.05$ considered significant).

\section{Conclusion and Future Work}

In this work, we demonstrated that using structured features boosts performance of supervised annotation learning. We proposed a formal annotation graph representation that can be used to derive these features automatically. However, the space of possible feature subgraphs can grow very large with more prior annotations. Standard feature selection techniques based on class association scores are less effective when the dataset is small. Feature voting from the user for identifying the relevant features is limited to simple features. Supplementary input from the user in terms of highlighted rationales can be used instead to prune the feature space. The proposed approach is general and can be applied to a variety of problems and features.

In this work, we presented our results with degree -0 and degree -1 feature subgraphs. We will extend our algorithm to automatically extract higher degree features from the annotation graph. For the rationale annotated training data $(R T D(i, j))$, we experimented with two possible values for $i$ and $j$. We aim to learn these weights empirically using a held out dataset. Rationales are associated with an additional cost per example and hence two approaches, with and without the rationales, are not directly comparable in terms of the number of examples. In the future, we will conduct an annotation experiment with real users to evaluate the usefulness of rationales in terms of clock time.

\section{Acknowledgments}

We would like to thank Dr. Carolyn P. Rose for her help with statistical analysis of the results. We would also like to thank all the anonymous reviewers for their helpful comments.

\section{References}

Cohen W. Minorthird: Methods for Identifying Names and Ontological Relations in Text using Heuristics for Inducing Regularities from Data. 2004. (http:// minorthird. sourceforge. net/).

Druck G., Mann G. and McCallum A. Learning from labeled features using generalized expectation criteria. In Proceedings of the ACM SIGIR, 2008.

Michael Gamon. 2004. Sentiment classification on customer feedback data: noisy data, large feature vectors, and the role of linguistic analysis. In Proceedings of COLING, 2005.

Haghighi A. and Klein D. Prototype-driven learning for sequence models. In Proceedings of the NAACL HLT 2006.

Minqing Hu and Bing Liu. 2004. Mining and Summarizing Customer Reviews. In Proc. of the ACM SIGKDD International Conference on Knowledge Discovery \& Data Mining.

Klein D. and Manning C. Accurate Unlexicalized Parsing. In Proceedings of ACL 2003.

Krovetz R. Viewing Morphology as an Inference Process. http://ciir.cs.umass.edu/ pubfiles/ir-35.pdf

Manning C., Raghavan P. and Schütze H. Introduction to Information Retrieval. Cambridge University Press. 2008.

Nenkova A. and Passonneau R. Evaluating Content Selection In Summarization: The Pyramid Method. In Proceedings of HLT-NAACL 2004.

Pang B. and Lee L. "A Sentimental Education: Sentiment Analysis Using Subjectivity Summarization Based on Minimum Cuts" In Proceedings of the ACL, 2004.

Sameer S. Pradhan, Wayne Ward, Kadri Hacioglu, James H. Martin, Daniel Jurafsky. 2004. Shallow Semantic Parsing using Support Vector Machines. In Proceedings of HLT/NAACL-2004,Boston, MA, May 27, 2004

Raghavan H., Madani O. and Jones R. Active Learning with Feedback on Both Features and Instances. Journal of Machine Learning Research, 2006.

Toutanova K. and Manning C. Enriching the Knowledge Sources Used in a Maximum Entropy Part-of-Speech Tagger. In Proceedings of EMNLP/VLC-2000.

Zaidan O., Eisner J. and Piatko C. Using "annotator rationales" to improve machine learning for text categorization. In Proceedings of NAACL-HLT 2007.

Zaidan O. and Eisner J. Modeling Annotators: A Generative Approach to Learning from Annotator Rationales. In Proceedings of EMNLP 2008. 